\section{UK's New CJD Not Detected in US}

Creutzfeldt-Jakob disease (CJD) in humans and bovine spongiform encephalopathy (BSE) in cattle are subacute degenerative diseases of the brain classified as transmissible spongiform encephalopathies. BSE was identified first in 1986 in the United Kingdom, where an epizootic involving over 155,000 cattle appeared to have been amplified greatly by exposure of calves to contaminated rendered cattle carcasses in the form of meat and bone meal nutritional supplements. In March 1996, an expert advisory committee to the UK government announced its conclusion that the agent responsible for BSE might have spread to humans, based on recognition of 10 persons with onset of a reportedly new variant form of CJD between February 1994 and October 1995. The 10 persons ranged in age from 16 to 39 years. In comparison, in the United States, deaths associated with CJD among persons under 30 have been rare, with median age at death being 68 years. As a result of this newly recognized variant of CJD in the UK, the CDC updated its previous review of national CJD mortality data. These reviews did not detect evidence of the occurrence of the newly described variant form of CJD in the United States. To broaden surveillance efforts in the United States, the CDC is encouraging physicians to increase their index of suspicion for this illness and, with state and territorial epidemiologists, is investigating CJD deaths among persons aged $<55$ years identified through routinely reported mortality data. Ongoing CJD and BSE surveillance in many countries of the world, including the United States, and especially the UK, will be critical for determining whether, and to what extent, the agent of BSE is causing disease in humans. This need is underscored by reports in 1996 of two additional confirmed cases of the newly recognized variant of CJD in persons with onset at age $<30$ years, one in France and one in the United Kingdom.

FROM: Centers for Disease Control and Prevention. Surveillance for Creutzfeldt-Jakob disease-United States. MMWR 1996;45(31):665-668.

\section{Laboratory Outbreak of Shigella}

Dr. Leonard Mermel and colleagues from Rhode Island Hospital recently reported an outbreak of Shigella sonnei in a university-affiliated hospital microbiology laboratory. During a 6-day period, January 23-28, 1996, 6 of 20 medical technologists in the microbiology laboratory became ill with stool-culture-confirmed Shigella sonnei sharing the same antibiogram. A medical technology student had been given an $S$ sonnei as an unknown pathogen to identify on January 16. Unlike other technologists in the laboratory, he routinely wore gloves for specimen handling and recalls gross contamination of his gloves during processing of the $S$ Sonnei specimen; he did not develop $S$ sonne $i$ infection. All the cases involved technologists working in the main bacteriology section of the microbiology laboratory, where one sink with handles for hot and cold water is used for handwashing.
Environmental cultures were negative for $S$ sonnei. In a case-control study, the one significant risk factor was touching faucet handles upon completion of handwashing without using a clean paper towel to turn off the water. No additional cases occurred following thorough cleaning of sink and the installation of a handle-free sink.

FROM: Mermel LA, Dempsey J, Parentesu S, Perry C, Josephson S. An outbreak of Shigella sonnei in a microbiology laboratory. Presented at the 36th Interscience Conference on Antimicrobial and Chemotherapeutic Agents; September 1996; New Orleans, LA. Abstract J109.

\section{Prevention of Nosocomial Pneumonia in NNIS Hospitals}

Nosocomial pneumonia remains a major cause of morbidity and mortality in US hospitals, and the CDC has published guidelines for the prevention of nosocomial pneumonia. To determine the infection control practices used to prevent nosocomial pneumonia, in February 1995, the CDC mailed a questionnaire to all of the hospitals participating in the CDC's National Nosocomial Infection Surveillance (NNIS) System. The survey assessed compliance with recommendations from the 1994 CDC Guidelines for the Prevention of Nosocomial Pneumonia and the use of other practices for which there are no specific recommendations from the CDC. Of the 188 surveyed hospitals, 179 (96\%) returned the survey. Of these, 166 (93\%) had implemented the recommended changing of the breathing circuit at $>48$-hour intervals. Of the 98 hospitals using humidifiers with mechanical ventilators, 96 (98\%) used sterile water to fill humidifiers. Other practices for which there are no specific CDC recommendations, and their frequency of use by NNIS hospitals, include the use of hygroscopic condenser humidifiers $(\mathrm{HCH})$ or heat-moisture exchanges (HME) with ventilators (110 of 179 [92\%]); routine change of $\mathrm{HCH}$ or HME (102 of 110 [93\%]); the use of a bacterial filter in the anesthesia machine (128 of 179 [72\%]); use of a multi-use closed catheter system during suctioning of patients on mechanical ventilation (156 of 179 [87\%]); or use of a single-use, open catheter system when suctioning patients not on mechanical ventilators (163 of 179 [92\%]). The authors concluded that most NNIS hospitals were practicing specific recommendations from the 1994 CDC Guidelines, as well as other infection control practices for which no specific recommendations are provided. However, further studies are needed to assess the impact of these practices on the occurrence of nosocomial pneumonia.

FROM: Managan LP, Banerjee SN, Tablan O, Simonds D, Jarvis WR. Infection control practices used for the prevention of nosocomial pneumonia by hospitals in the National Nosocomial Infections Surveillance (NNIS) System. Presented at the 36th Interscience Conference on Antimicrobial and Chemotherapeutic Agents; September 1996; New Orleans, LA. Abstract J74. 